\title{
Géolinguistique
}

$17 \mid 2017$

Varia

\section{Enregistrements et transcriptions pour un atlas sonore des langues régionales de France}

Recordings and Transcriptions for a Speaking Atlas of the Regional Languages

of France

Philippe Boula de Mareüil, Frédéric Vernier et Albert Rilliard

\section{(2) OpenEdition}

Journals

Édition électronique

URL : http://journals.openedition.org/geolinguistique/365

DOI : 10.4000/geolinguistique.365

ISSN : 2650-8176

Éditeur

UGA Éditions/Université Grenoble Alpes

Édition imprimée

Date de publication : 1 décembre 2017

Pagination : 23-48

ISBN : 978-2-37747-025-9

ISSN : 0761-9081

\section{Référence électronique}

Philippe Boula de Mareüil, Frédéric Vernier et Albert Rilliard, « Enregistrements et transcriptions pour un atlas sonore des langues régionales de France », Géolinguistique [En ligne], 17 | 2017, mis en ligne le 01 février 2019, consulté le 01 novembre 2020. URL : http://journals.openedition.org/geolinguistique/ 365 ; DOI : https://doi.org/10.4000/geolinguistique.365 


\title{
Enregistrements et transcriptions pour un atlas sonore des langues régionales de France
}

\author{
Philippe Boula de Mareüil \\ Univ. Paris-Saclay, LIMSI-CNRS \\ Frédéric Vernier \\ Univ. Paris-Saclay, LIMSI-CNRS
}

Albert Rilliard

Univ. Paris-Saclay, LIMSI-CNRS

Univ. Federal de Rio de Janeiro

\section{Résumé}

L'objectif est de montrer et de valoriser la diversité linguistique de la France, à travers des enregistrements recueillis sur le terrain, une réalisation informatique (qui permet de visualiser les aires dialectales) et un travail de transcription orthographique (qui représente un objet de recherche en soi). Il y a ainsi une dimension à la fois scientifique et patrimoniale à ce travail, dans la mesure où un certain nombre de langues régionales ou minoritaires sont en situation critique.

\section{Mots-clés}

Atlas sonore, langues de France.

\section{Abstract}

The aim of this paper is to show and promote the linguistic diversity of France, through field recordings, a computer achievement (which allows us to visualise dialectal areas) and an orthographic transcription work 
(which represents an object of research in itself). There is thus both a scientific dimension and a heritage dimension in this work, insofar as a number of regional or minority languages are in a critical situation.

\section{Keywords}

Speaking atlas, languages of France.

\section{Introduction}

Même si dans l'Occident moderne de grandes langues de diffusion ont réussi à s'imposer, le dialectologue observe vite sur le terrain que la diversité est première. L'idée de reporter cette diversité sur des cartes n'est pas nouvelle (Le Dû, Le Berre \& Brun-Trigaud, 2005) : de 1897 à 1901, E. Edmont a sillonné la France et sa périphérie gallo-romanes pour mener des enquêtes dans plus de 600 communes, cartographiées dans l'Atlas linguistique de la France [ALF] (Gilliéron \& Edmont, 1902-1910). Entre 1911 et 1914, F. Brunot a lancé le projet d'un atlas linguistique phonographique de la France, mais celui-ci est resté à l'état d'ébauche, avec seulement trois enquêtes dans les Ardennes, le Berry et le Limousin ${ }^{1}$. À partir de la Seconde Guerre mondiale, les Atlas linguistiques de la France par régions (Séguy, 1973a; Tuaillon, 1976) ont poursuivi la documentation de langues et de dialectes de plus en plus menacés - la limite entre langues et dialectes n'étant pas définie de manière univoque.

Récemment, des enregistrements audio ont été numérisés, au moins pour le domaine occitan, dans le cadre du Thesaurus occitan [Thesoc] (Sauzet \& Brun-Trigaud, 2013) et le domaine francoprovençal, avec l'Atlas multimédia de la région Rhône-Alpes [ALMuRA] ${ }^{2}$ (Médélice, 2008), mais ils ne sont pas complètement disponibles - et le domaine d'oïl est encore moins bien loti (Léonard \& Djordjević, 2009). Reprenant le principe des atlas dialectologiques sur papier et tirant bénéfice de l'outil informatique, le Corpus de la parole donne aujourd'hui accès en ligne à des fonds sonores d'une grande richesse (Jacobson \& Baude, 2011).

1. <http://gallica.bnf.fr/html/und/enregistrements-sonores/archives-de-la-paroleferdinand-brunot-1911-1914>.

2. <www.atlas-almura.net>. Ce site fait suite aux travaux de I. Marquet (1995), dont l'Atlas parlant des Alpes n'est aujourd'hui plus visible sur Internet. 
Cependant, les données comparables d'une région à l'autre font cruellement défaut, et malgré des projets récents ${ }^{3}$, la France est dans ce domaine quelque peu à la traîne par rapport aux Pays-Bas et à la Norvège (Heeringa, 2004), à l'Allemagne et à l'Italie (Iannàccaro \& Dell'Aquila, 2001) ${ }^{4}$, par exemple. La place prépondérante de la langue française masque une diversité linguistique parmi les plus exemplaires d'Europe, que la Charte européenne des langues régionales ou minoritaires (signée par la France en 1999, mais non ratifiée) se proposait de promouvoir ${ }^{5}$. De même, l'Unesco insiste sur la nécessité du multilinguisme dans le cyberespace (Vannin \& Le Crosnier, 2012); et des sites à orientation grand public se multiplient, proposant à des gens de s'enregistrer un peu partout sur le globe et à des réseaux sociaux de voter pour tel ou tel, mais sans contrôle linguistique ${ }^{6}$. Il est donc important, voire urgent, de recueillir des enregistrements suivant une approche coordonnée, appliquant un protocole commun pour donner une meilleure image de la pluralité des usages.

Il y a encore quelques décennies, il aurait sans doute été plus aisé de trouver des locuteurs de dialectes et langues régionales en France. L'Internet qui permet aujourd'hui d'entrer en contact avec nombre d'associations de promotion de langues minoritaires était cependant moins développé à l'époque, de même que les moyens de collecter et de stocker des enregistrements en grandes quantités. Alors que la plupart de ces dialectes et langues sont désormais en danger, c'est un atlas linguistique sonore que nous décrivons ici. Cet atlas prend la forme d'un site web présentant une carte de France interactive (pour l'instant limitée à la Métropole, même si des enregistrements ont également été collectés dans les cinq départements-régions d'Outre-mer), avec plus d'une centaine de points d'enquête sur lesquels l'internaute peut cliquer pour entendre autant d'échantillons de parole et lire une transcription de ce qui est dit. La question de la transcription orthographique des langues régionales de France continue à faire débat : représenter les sons de la parole par des signes graphiques n'étant jamais sans poser de problèmes, nous nous y arrêterons donc.

3. Voir p. ex. <http://symila.univ-tlse2.fr/>.

4. Voir aussi, p. ex., <www.hf.ntnu.no/nos/>, <http://atlantelinguisticosicilia.it/cms/ home/cartasonora/s.

5. «Les langues régionales appartiennent au patrimoine de la France», stipule l'article 75-1 de la Constitution de la Cinquième République française. Elles sont quelque 75 inventoriées dans le rapport Cerquiglini (<www.ladocumentationfrancaise.fr/var/ storage/rapports-publics/994000719.pdf>), dont la majorité dans les Outre-mer. Il ne s'agit pas ici d'entamer une discussion sur la portée de ces dispositions légales, mais on pourra consulter à ce sujet, par exemple, Costaouec (2013).

6. Voir, entre autres, <https://localingual.com/>. 
En l'absence de traditions écrites bien établies et d'une autorité unique, les langues régionales de France ne bénéficient pas de standards reconnus et acceptés par tous (Caubet, Chaker \& Sibille, 2002; Vannini \& Le Crosnier, 2012). Dès lors, les solutions proposées varient selon les langues, même parmi les langues territoriales de France, qui sont fondées sur l'alphabet latin et se veulent un minimum (ou le plus possible) fidèles à la prononciation. Les graphies adoptées sont plus ou moins phonétiques (reflétant une prononciation locale particulière) ou diasystémiques (mettant en avant l'unité d'un ensemble de dialectes). Parfois, le système est hybride, notant ce qui dans la prononciation se distingue du français, tout en suivant les conventions orthographiques du français (ex. voéyageux). Ces systèmes ont chacun leurs avantages et leurs inconvénients.

Ces différents types de graphie ont été mis à l'épreuve à travers un cas d'école : la transcription d'un même texte d'une centaine de mots, que nous commencerons par présenter. Nous décrirons ensuite les enquêtes que nous avons menées, les locuteurs dont nous avons collecté et transcrit des enregistrements, le protocole appliqué et la cartographie établie. Nous présenterons enfin une première analyse des données recueillies.

\section{Pour un atlas sonore des langues régionales de France}

\subsection{Points d'enquête et locuteurs}

Le matériau sur lequel nous nous sommes focalisés est constitué de la fable d'Ésope «La bise et le soleil» (120 mots en français, environ une minute de parole), traduite et enregistrées dans plus d'une centaine de points d'enquête dans autant de variétés de langues régionales de France. Avec ce texte, utilisé depuis plus d'un siècle par l'Association phonétique internationale (API) pour décrire un grand nombre de langues et dialectes du monde, on renouait ainsi de façon laïque avec une tradition remontant au XIX ${ }^{\mathrm{e}}$ siècle, consistant à faire traduire en langues locales la parabole de l'enfant prodigue. Le corpus recueilli a donné lieu à une réalisation informatique couvrant des langues romanes (d'oïl, d'oc, mais aussi le francoprovençal, le catalan et le corse), des langues germaniques (l'alsacien, le francique ${ }^{7}$ et le flamand occidental), ainsi que le breton (langue celtique) et le basque.

Pour rencontrer ces locuteurs, nous avons contacté des universités et des radios locales, des associations et offices publics de promotion de langues

7. Ce glossonyme est le nom scientifique de ce que la plupart des Lorrains appellent «platt» (Rispail, 2014). 
régionales et des cercles de patoisants, des mairies, des offices du tourisme et des écoles immersives (diwan, calendretas). Un temps considérable a été passé pour trouver des locuteurs de langues aujourd'hui très minoritaires, organiser et optimiser les déplacements. Cette recherche a nécessité de consulter une importante documentation non seulement sur les dialectes mais également sur l'histoire et la géographie des régions explorées. En 2014-2016, nous avons mené une trentaine d'enquêtes de terrain, à raison de quelques jours par mission, cherchant à maximiser le nombre de locuteurs par localité.

Nous avons ensuite sélectionné un locuteur par localité, ne retenant qu'une seule commune dans un rayon de moins de $20 \mathrm{~km}$. Nous avons privilégié les locuteurs natifs, mais n'avons pas dédaigné les néo-locuteurs, dans des villes comme Bordeaux ou Nice. Dans certains cas, il n'y avait guère le choix; dans d'autres, un des locuteurs se distinguait par ses talents oratoires, le caractère esthétique de sa voix ou son ancrage local. La distinction entre «néo-locuteurs» et «locuteurs naturels» n'est d'ailleurs pas toujours évidente (Léonard \& Jagueneau, 2013) : il existe des locuteurs natifs de néo-breton, par exemple; des locuteurs dits «naturels» peuvent avoir une langue régionale très francisée; au contraire, certains locuteurs qui n'ont pas hérité du dialecte directement de leurs parents peuvent s'appliquer avec succès à reproduire les particularismes locaux. Quand un locuteur est né et a toujours vécu en un même lieu, associer son enregistrement à un point sur la carte est aisé, mais la vie moderne incite à être mobile. Quand un locuteur déclarait explicitement parler le dialecte d'un endroit qui n'était pas son lieu de résidence, nous avons retenu cet endroit pour le lui associer - le plus souvent la commune où le locuteur avait grandi.

Les locuteurs, assez engagés sur le terrain culturel et linguistique, étaient de milieux socio-professionnels variés. Un profil typique était le suivant : un homme retraité, issu du monde paysan (ou ouvrier, dans le Nord et l'Est), ayant connu une ascension sociale, ayant par exemple travaillé comme enseignant. À noter que dans l'ALF, également, E. Edmont a enquêté majoritairement auprès d'hommes instruits, en moyenne plus âgés dans le Nord que dans le Sud. Tous ceux qui nous ont répondu positivement nous ont réservé un accueil extrêmement chaleureux, exprimant souvent le sentiment émouvant que leur «patois » allait s'éteindre avec eux, dans le domaine d'oïl et le Croissant (zone intermédiaire entre celles d'oïl et d'oc, ainsi nommée à cause de sa forme). Ils montraient en général, sans nécessairement être des «érudits locaux», une connaissance très fine de la culture, de l'histoire et de la géographie de leur région, rendant les échanges très enrichissants. Ils ont pour la plupart été enregistrés à leur domicile ou dans une salle de réunion (accueillant des ateliers de patoisants, 
par exemple), avec un enregistreur ZoomH4 au format Wave (en stéréo, avec une fréquence d'échantillonnage de $44,1 \mathrm{kHz}$ et une résolution de 16 bits, avant égalisation de l'intensité et conversion en MP3 pour la mise en ligne). Chaque enregistrement était associé à un consentement signé pour une libre diffusion.

\subsection{Protocole et transcription}

Un protocole commun a été appliqué, dans lequel il était d'abord demandé aux locuteurs de lire la fable en français. Il leur était ensuite demandé de traduire ce texte dans leur langue régionale, soit directement avec le texte français sous les yeux, soit à partir de notes que les locuteurs avaient préféré prendre. Certains locuteurs (notamment au Pays basque, en Bretagne, en Flandre occidentale et dans le domaine d'oïl) ont tenu à écrire entièrement leurs traductions. Ce cas de figure s'est produit soit parce que la langue régionale et le français étaient très différentes dans leurs structures (avec un ordre des mots éventuellement différent, comme en breton), soit parce qu'au contraire la langue régionale était proche du français, comme dans le domaine d'oïl, et que les locuteurs avaient besoin de se «remettre dans le bain».

Le français, en France, domine tant dans les interactions quotidiennes et l'espace public que la tâche de traduction n'est pas aisée. Il reste qu'en corse, en catalan, en occitan, en francoprovençal, en alsacien et parfois dans le domaine d'oïl, les locuteurs que nous avons enregistrés sont fort bien parvenus à traduire la fable d'Ésope à la volée. Un mot comme «voyageur» a pu faire difficulté, parce qu'à l'époque où on parlait «patois », nous disaient certains, on ne voyageait pas. Toujours est-il que traduire des fables de La Fontaine ou autres était un exercice auquel plusieurs de nos informateurs étaient rôdés. Parfois les locuteurs - des conteurs, en gallo, en normand, en mainiot, en poitevin-saintongeais, en welche (lorrain), en franc-comtois ou en francique, par exemple - se sont éloignés d'une traduction littérale pour se rapprocher de leurs traditions orales. Ces différentes stratégies de traduction sont également un témoignage de richesse, de diversité.

Pour l'occitan (langue d'oc), nous avons transcrit les enregistrements en graphie classique - en nous inspirant parfois de ce que les locuteurs nous avaient fourni - et avons fait vérifier et éventuellement corrigé les transcriptions par des spécialistes, qui avaient également accès à l'audio ${ }^{8}$.

8. Seul pour un point de Provence, Sanary-sur-Mer, nous sommes restés fidèles à la graphie mistralienne à laquelle le locuteur était attaché. Cette graphie est plus pho- 
Pour le catalan et le corse, de même, nous nous sommes fait aider pour vérifier/corriger les transcriptions que nous avions produites.

Les orthographes unifiées du basque (batua) et du breton (peurunvan) étaient bien connues de nos informateurs, respectivement bascophones et bretonnants. Pour l'alsacien, un système souple proche d'ORTHAL (Zeidler \& Crévenat-Werner, 2008) a été utilisé, avec pour référence graphique l'allemand écrit (Schriftsprache), dans le respect des particularités dialectales. Pour le francique luxembourgeois (parlé dans notre point d'enquête à Apach), c'est le standard luxembourgeois qui a été suivi, alors que pour les dialectes mosellans et rhénans ce sont d'autres systèmes qui ont été adoptés, également inspirés de la graphie de l'allemand moderne, mais faisant usage du digraphe ' $\mathfrak{~}$ ' et d'accents graves pour indiquer/surcaractériser le timbre ouvert de certaines voyelles, ainsi que de consonnes comme 'd' pour noter des occlusives sourdes non aspirées, comme [t] (Hudlett, 2004).

Dans le domaine d'oïl, en revanche, on a parfois affaire à des graphies individuelles, comme en berrichon (parmi les parlers centraux), en mainiot, en lorrain ou en wallon, pour lesquels en France il n'existe pas vraiment de standard. Ceci vaut également pour trois points d'enquête dans le Croissant (Pleuville, Bussière-Poitevine et Éguzon-Chantôme), pour lesquels une graphie francisante a été adoptée. Le scripteur écrit ainsi les mots tels qu'il voudrait qu'on les «entende» en respectant son phonétisme propre. Le plus souvent, c'est une orthographe francisée qui s'est imposée; ce qu'écrivait Contejean (1876) à propos du franc-comtois peut s'appliquer à d'autres «patois » : «L'orthographe du patois doit se rapprocher autant que possible de l'orthographe du français, en raison de l'intime analogie des deux langues.» Les règles édictées par cet auteur valent également pour les transcriptions que nous ont fournies nos principaux informateurs pour des variétés d'angevin, de bourguignon, de champenois, de normand et de picard:

- conservation de la marque du pluriel pour les noms, pronoms et adjectifs (en général le $s$ );

- conservation de la marque des personnes pour les conjugaisons;

- souci de clarté, évitant d'accumuler les élisions à travers des apostrophes - marqueurs d'une oralité considérée comme inhérente aux «patois».

nétique que la graphie dite «classique» ou «alibertine» (Alibert, 1935; Sumien, 2007). Le visiteur du site peut ainsi avoir un aperçu de la pluralité des graphies. 
Le système dit Feller-Carton, pour le picard (Carton, 2009), propose de même deux principes, assortis de règles par sous-régions pour résoudre les cas où ces deux principes sont en contradiction :

- priorité à la graphie française sous réserve qu'elle ne crée pas d'équivoque;

- priorité à la phonétique du picard.

Le tableau 1 consigne certaines des directives dont les locuteurs se sont inspirés, plus ou moins fidèlement. Nous verrons plus loin comment ou s'ils ont résolu de possibles contradictions. Dans le domaine d'oïl, l'expérience montre par exemple que proscrire le ' $\mathrm{x}$ ' pour orthographier le normand est source d'hésitation dans le cas de mots comme deux. De même, imposer en francoprovençal d'écrire bize pour traduire le français «bise» est source d'erreur pour des scripteurs alphabétisés en français (cf. infra).

\begin{tabular}{|l|l|}
\hline alsacien & $\begin{array}{l}\text { Académie pour une graphie alsacienne } \\
\text { transfrontalière (AGATe) }\end{array}$ \\
angevin & Cercle Jules Ferry \\
bourguignon-morvandiau & Langues de Bourgogne \\
flamand occidental & Lou Champaignat \\
franc-comtois & Akademie voor Nuuze Vlaemsche Taele \\
francoprovençal & Contejean \\
francique & Groupe de Conflans \\
gallo & Gau un Griis, médiathèque de Sarreguemines \\
normand & Auffray, Bienvenu, Le Coq, Dréano (ABCD) \\
picard & Lechanteur \\
poitevin-saintongeais & Feller-Carton \\
& Union pour la culture populaire en Poitou- \\
\hline
\end{tabular}

Tableau 1. - Origines de quelques normes de transcriptions de langues régionales de France (associations ou personnes).

Dans tous les cas, nous avons veillé à l'adéquation entre ce qui était dit et ce qui était transcrit, car on a vite fait de ne pas écrire exactement les mots qui sont prononcés et de ne pas lire exactement les mots qui sont manuscrits ou imprimés. Quand cela était nécessaire, nous avons supprimé dans le signal audio les disfluences et aparté : il aurait été malvenu, en effet, de laisser des commentaires (du type es pas aisit, «ce n'est pas facile» en occitan) en plein milieu de la fable. 


\subsection{Cartographie}

Les points d'enquête réalisés, définis par leur latitude et leur longitude, ont été disposés sur une carte de France où nous avons fait figurer, en plus des frontières de départements qui permettent de délimiter les régions administratives, les limites entre domaines linguistiques. Ces dernières sont éminemment plus discutables et généralement moins abruptes; G. Paris (1888) rejetait la notion même de frontière dialectale, voire la séparation entre langues d'oïl et d'oc que certains cherchaient à localiser. Sa vision continuiste était du reste partagée par son élève J. Gilliéron, qui s'intéressait à l'histoire des mots plus qu'à celle des patois, «cette unité artificielle, impure et suspecte» (Gilliéron \& Mongin, $1905: 27)$. Si en effet on regarde des isoglosses tracées selon des critères différents, elles peuvent ne pas coïncider, et il est alors difficile de déterminer lesquelles utiliser de préférence. On sait aussi que le critère d'intercompréhension, parfois avancé, trouve rapidement ses limites : question de degré et source d'enjeux identitaires, il peut se montrer asymétrique et dépendre des situations de communication. Toute classification étant sujette à caution (Goebl, 2002; Gaillard-Corvaglia, Léonard \& Darlu, 2007; Sumien, 2012), celle que nous proposons n'a aucune prétention à être définitive. Elle retient 25 langues régionales ou dialectes primaires, regroupés comme indiqué dans la figure 1 .

Nous avons repris une partition classique en langues romanes (d'oïl, d'oc, catalan, francoprovençal et corse ${ }^{9}$ ), langues germaniques (alsacien, flamand occidental, francique) et «autres langues» (basque et breton). Ces dernières langues, sur le territoire français, sont traditionnellement sousdivisées en dialectes : luxembourgeois, mosellan et rhénan pour le francique, labourdin, bas-navarrais et souletin pour le basque; trégorrois, léonard, cornouaillais et vannetais pour le breton. Même si chacun de ces dix dialectes est représenté par un point d'enquête, nous n'avons pas fait figurer ces étiquettes sur la carte, pour des raisons d'échelle.

Il se trouve que la majorité des associations de défense et de promotion des langues d'oïl et d'oc insistent sur le pluriel dans le premier cas («les langues d'oïl») et sur le singulier dans le second («la langue d'oc», une et diverse), même si le gascon est incontestablement assez distinct des autres variétés d'oc (Chambon \& Greub, 2002). Ces deux conceptions étant difficilement conciliables (Abalin, 2007), par respect pour nos informateurs

9. Contrairement aux langues précédentes, le corse s'inscrit dans l'aire italo-romane, avec quelques éléments gallo-romans (Medori, 2013). Nous n'avons cependant pas fait apparaître cette classification dans la légende. 
d'oïl et d'oc, nous n'avons pas tranché : nous avons indiqué dans la légende «langue(s) d'oïl» et «langue d'oc (occitan)». Des couleurs chaudes (dans les rouges) ont été choisies pour les variétés d'oc, des couleurs froides (dans les bleus) pour les variétés d'oïl, tandis que le vert a été retenu pour le francoprovençal et des nuances de jaunes pour les langues germaniques. Des traits plus épais soulignent les limites entre ces différents domaines, tandis que les limites du Croissant, apparaissent floutées, pour suggérer le caractère transitoire de cette aire linguistique.

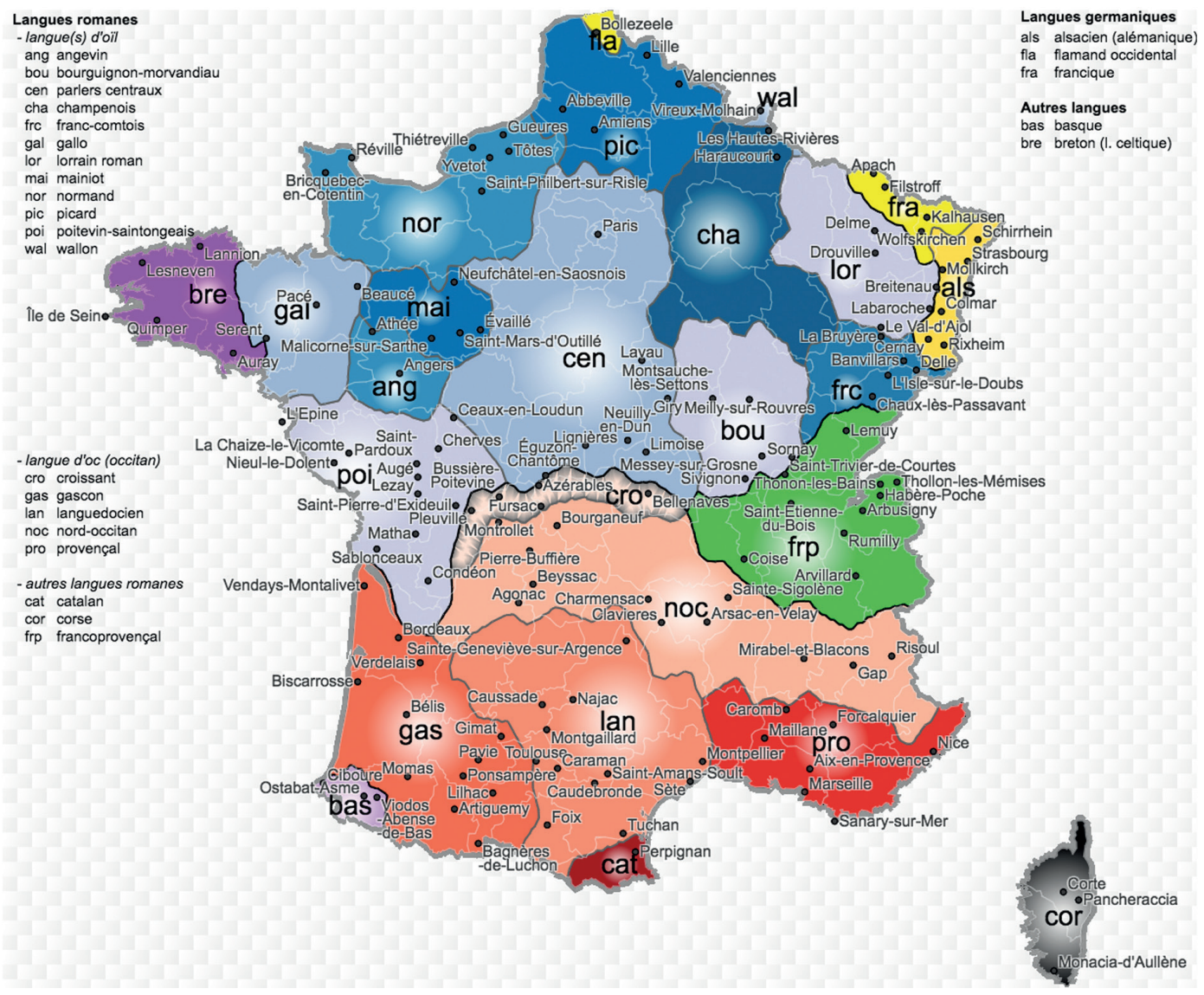

Figure 1. - Interface du site <https://atlas.limsi.fr $>$. 
Pour tracer ces limites linguistiques, nous avons classifié les quelque 600 points d'enquête de l'ALF dans nos 25 catégories, en nous appuyant sur divers atlas régionaux et publications : pour le domaine d'oïl (Bourcelot, 1966-1978; Brasseur, 1980-2011 ; Carton \& Lebègue, 1989-1997; Dondaine, 1972-1978; Dubuisson, 1971-1982; Guillaume \& Chauveau, 1975-1983; Lanher, Litaize \& Richard, 1979-1988; Massignon \& Horiot, 1971-1983; Simoni-Aurembou, 1973-1978; Taverdet, 1975-1980; Martin, 2015); pour le domaine francoprovençal (Gardette, 1952; Tuaillon \& Contini, 1996); pour le domaine d'oc (Séguy, 1973a, 1973b; Desrozier \& Ros, 1974 ; Sumien, 2008). Ce dernier domaine linguistique a été subdivisé, suivant Tuaillon et Contini (1996) en : gascon, languedocien, provençal, nord-occitan et Croissant. Nous aurions pu subdiviser le sousdomaine nord-occitan (et le Croissant) en limousin, auvergnat et vivaroalpin. Cependant, plus on multiplie les frontières plus on crée de problèmes : les locuteurs vellaves (du Velay), par exemple, sont difficiles à classer. Faire figurer le Croissant, en revanche, montre bien la forme de cet ensemble de parlers de transition entre oïl et oc. Quant au domaine des parlers centraux d'oïl, nous n'avons pas cherché à le subdiviser en tourangeau, orléanais/solognot, etc., faute de locuteurs actuels de ces dialectes.

Un algorithme a ensuite été conçu, pour tracer des lignes passant au milieu des deux points de catégories différentes, lisser les contours et colorer les zones ainsi définies. Le lissage des limites entre aires dialectales, plus ou moins en dents de scie, peut être réglé par l'utilisateur, à l'aide d'un curseur. Pour affiner les tracés, des points ont été ajoutés aux alentours des frontières linguistiques des domaines basque, breton, catalan, alsacien, flamand et francique, de même qu'aux confluents des aires gasconne, languedocienne et nord-occitane. Par ailleurs, un champ «Recherche» est offert à l'utilisateur, et des options ont été prévues pour afficher ou non les frontières de départements, les points ALF et nos points d'enquête, enfin Jersey, la Suisse romande et la Belgique francophone d'une part, l'Outremer d'autre part.

La carte interactive qui en résulte, avec ses plus de 140 points sur lesquels on peut cliquer pour entendre autant de versions d'un même texte, la fable d'Ésope ${ }^{10}$ «La bise et le soleil», est accessible en ligne à l'adresse $<$ https://atlas.limsi.fr> (en français et en anglais). Cette fable peut être écoutée (et lue) en français, en cliquant sur Paris à cette adresse.

10. Cette fable XLVI, mise en vers par La Fontaine sous le titre «Phébus et Borée», commence ainsi dans la version en français moderne utilisée par l'API : «La bise et le soleil se disputaient.» 
Le site permet d'apprécier immédiatement combien certains parlers voisins géographiquement diffèrent linguistiquement. Il en va ainsi de points d'enquête en Bresse bourguignonne et en Bresse savoyarde (francoprovençale), de points en Alsace alémanique et en Alsace romane (welche), et a fortiori de points du Pays basque et du Béarn (gascon), rassemblés dans le département des Pyrénées-Atlantiques. C'est aux points communs entre divers parlers que nous nous attacherons dans la section suivante. En creux, par rapport au maillage du territoire, il est frappant, même si cela n'est pas étonnant, qu'aucun locuteur de dialecte n'ait été trouvé autour de Paris : pour les parlers centraux, nous n'en avons guère trouvé que dans le Berry, dans le Bourbonnais et vers le Nivernais. À l'Est, surtout, tout se passe comme si les dialectes avaient été relégués aux frontières belge, allemande et suisse. Nous n'avons trouvé des locuteurs de champenois et de lorrain, pratiquement, que dans les Ardennes et dans les Vosges, respectivement. L'annexion de la Moselle par l'Allemagne a pu donner quelques répits aux dialectes (y compris romans, qui n'étaient pas interdits comme le français), mais la locutrice que nous avons pu enregistrer dans ce département fait aujourd'hui figure d'exception.

\section{Analyse des enregistrements et des transcriptions}

Certains traits linguistiques se retrouvent dans différents domaines, à différents niveaux. Commençons par les niveaux phonético-phonologiques.

\subsection{Prononciation}

Au niveau phonétique, le /k/ du latin a pu se maintenir en corse, en catalan, en sud-occitan ainsi qu'en normand, au nord de ce qu'il est convenu d'appeler la ligne Joret ${ }^{11}$ et en picard (d'où des formes comme récauf(f)é). Ce $/ \mathrm{k} /$ a pu évoluer en $[\mathrm{S}]$ autour des parlers centraux (d'où des formes comme réchauffé) en une interdentale $[\theta]$ (notée «sh») en francoprovençal (d'où des formes comme réshodô) et s'affriquer en [ts] en nord-occitan comme dans le domaine «tseu» de Bourgogne (d'où des formes comme rétsindu). Un autre trait que l'on note dans le Sud et dans la périphérie Nord-Est, dans la pointe de Givet de dialecte wallon, est la présence d'un /s/ dans des formes verbales comme estar, rescalfat (catalan et occitan), estant,

11. Cette ligne ainsi nommée, à la suite des travaux de Joret (1881), est un faisceau d'isoglosses permettant de distinguer les parlers normano-picards du reste du domaine d'oïl. 
restchauffé (wallon). Perceptivement très saillant, un [h] peut s'entendre non seulement dans les langues non romanes mais aussi dans la plupart des variétés de normand (où le phonème correspondant est noté « $\underline{\mathrm{h}}$ », par exemple dans hardes), de poitevin-saintongeais (où le phonème correspondant, noté «jh», correspond au /3/ français, par exemple dans voeyajheur) et de gascon (où le phonème correspondant, noté «h», provient de la laryngalisation du /f/ latin, par exemple dans hòrt). De même, un [ç] s'entend en Bresse bourguignonne (transcrit $\left\langle\mathrm{sc}^{\prime}\right\rangle$, ex. sousc'iller «souffler») en franc-comtois près de la frontière suisse (transcrit «çh», ex. çhioûçhaie «souffler»), en Poitou (également transcrit «çh», ex. le démonstratif çhàu «celui») et en Alsace bossue (noté «ch» comme en allemand, même si le dialecte parlé dans le point d'enquête de Wolfskirchen n'est pas alémanique mais francique rhénan). Un $[\mathrm{x}]$ peut par ailleurs s'entendre en breton et dans les langues ou dialectes germaniques comme l'alsacien (également transcrit «ch» après une voyelle comme «o»), ainsi qu'en lorrain (transcrit «hh»), dans les Vosges. D'autre part, un [r] apical peut s'entendre dans tout le Sud, ainsi qu'en Bourgogne et dans le Maine. Apical ou non, le /R/ peut être l'objet de métathèse dans des formes comme arnoncit (poitevin-saintongeais) ou a arnonché (picard et normand). Quant au /1/, il peut être palatalisé en [j] après consonne, un yod qu'il a été proposé de noter «ll» en poitevin-saintongeais (ex. obllijhës), en normand et en bourguignon. Dans le domaine occitan, la vocalisation du /l/ en position de coda qu'on observe largement en provençal (par exemple dans $a u$, languedocien moyen $a l$ ) se retrouve en gascon, en nord-occitan ainsi qu'à Montpellier et à Sète (languedocien oriental). Toujours dans le domaine occitan, le bêtacisme (confusion entre $[\mathrm{b}]$ et $[\beta]$ ou $[\mathrm{v}]$ ) se retrouve en gascon, en languedocien ainsi que sporadiquement ailleurs, et est partagé avec le catalan (ex. arribaria « arriverait»). Le rhotacisme, lui, se retrouve en gascon, en nord-occitan et sporadiquement ailleurs dans le mot sorelh «soleil», où il peut également affecter la consonne finale $/ K /$, notamment en Auvergne. De plus, la prononciation [kwan] de quan est partagée par le gascon béarnais et le catalan.

$\mathrm{Au}$ niveau phonologique, les voyelles /œ/ et / $\varnothing / \mathrm{n}$ 'appartiennent pas au système de l'occitan, sauf peut-être en languedocien oriental et dans le nord du domaine avec le Croissant, mais se retrouvent dans tous les systèmes de la zone d'oïl. Concernant également les systèmes vocaliques, les diphtongues de type /ao/, caractéristiques du gallo (ex. paltaod) sont proches phonétiquement de ce qu'on peut entendre en poitevin-saintongeais (ex. paletàu) ou en normand. Au niveau prosodique, tous les parlers d'oïl sont oxytoniques, comme dans le Croissant, à la différence de ce qui se passe dans les autres langues. 
Parmi les langues romanes, certaines variétés connaissent même des proparoxytons : il en va ainsi du nissart (niçois, ex. la zísola, précisément «la bise») et du corse (ex. si litigavanu «se disputaient», même si dans ce dernier cas la place de l'accent n'est pas notée dans l'orthographe).

Au niveau morpho-phonologique, des infinitifs en /a/ pour les verbes du $1^{\text {er }}$ groupe sont les continuateurs du /a/ latin en corse, en catalan et en occitan, sauf dans certains endroits comme le Croissant bourbonnais, où l'on a un $/ \varepsilon /$. On retrouve ce $/ a /$, réduction de la diphtongue [ai] dans certains endroits du domaine poitevin-saintongeais, comme le Mellois (dans les Deux-Sèvres, ex. bufàe «souffler»), alors qu'on a [е:в] avec un /R/ qui s'est maintenu sur l'île de Noirmoutier, dans l'Atlantique (ex. bufér). Ce dernier fait est rarissime dans les langues gallo-romanes : seul dans les Alpes, autour de Gap, le -r final de l'occitan bofar [bufar] se fait entendre. Le suffixe agentif correspondant au -eur du français se ferme en [u] dans différents sous-domaines du bourguignon, du franc-comtois, du lorrain et du gallo (ex. vayaijou). Inversement, dans le domaine occitan, on a un [u] en gascon dans viatjador - avec une spécialisation du suffixe -ador (<-ATŌRE(M)), issu du cas régime dans la langue médiévale - alors qu'on note des gallicismes antériorisés en [y]/[œ] chez des locuteurs de différents sous-dialectes occitans. Dans ce suffixe très productif, par ailleurs, le $/ \mathrm{R} /$ tend à tomber chez la plupart des locuteurs de picard, du normand, du mainiot et de l'angevin. Concernant une autre terminaison, l'apparition d'un yod là où en français on a des mots en -eau (<-ELLum, ex. mantiau) s'observe en picard, en wallon, en champenois, en bourguignon, en angevin, en berrichon-bourbonnais et en poyaudin (parmi les parlers centraux), comme en saintongeais.

\subsection{Grammaire et lexique}

Au niveau morpho-syntaxique, des variantes de pi(s) pour traduire la conjonction de coordination «et»se retrouvent en francoprovençal, en bourguignon, en franc-comtois, en champenois, en lorrain, en picard, en normand, en poitevin-saintongeais et dans certains parlers centraux, tandis qu'adonc pour traduire le français «donc» se retrouve en wallon, en picard, en angevin et en occitan. On note également la forme c'ment pour la locution de subordination «comme» en franc-comtois, en bourguignon et en lorrain, ainsi que la locution conjonctive quand que (ou quand qu') dans des variétés d'angevin, de mainiot, de normand et des parlers centraux. L'article masculin défini est $e l$ en catalan ainsi que dans certaines variétés de picard et de normand. En normand, de plus, comme en gallo et en angevin, des variantes de le sien qui expriment le relatif français «celui qui ». 
Au niveau morpho-syntaxique, toujours, de l'expression de la possession, l'article défini est utilisé dans les possessifs en catalan (ex. el seu manto) et en corse (ex. u so mantellu), comme en gascon (ex. lo son manto) et en nissart (ex. lo sieu mantèu) pour traduire «son manteau». À noter toutefois que plusieurs variétés d'occitan préfèrent la formulation «s'est enlevé le manteau» à la tournure «a enlevé son manteau». Comme le catalan et le corse, la plupart des variétés occitanes sont pro-drop, mais le Croissant emploie un pronom personnel sujet (ex. ilhs à la $3^{\mathrm{e}}$ personne du pluriel). À cette personne, le pronom est $i l$, sans liaison devant voyelle, en francoprovençal, en champenois, en bourguignon, en mainiot et en gallo. Le plus souvent, ce pronom reprend anaphoriquement le syntagme sujet en angevin, en mainiot, en picard et en berrichon; il est transcrit de façon variable $\left(y, i, i^{\prime}, i s\right)$ devant consonne : on a par exemple le vent et l'soulei $y$ s'disputaient en mainiot. Au singulier, le pronom sujet de $3^{\mathrm{e}}$ personne féminin est alle en picard, dans la plupart des variétés de bourguignon et en angevin.

Au niveau de la morphologie flexionnelle, c'est principalement le système verbal qui appelle quelques remarques. Le passé simple est en usage dans tout le domaine occitan ainsi qu'en welche, en bourguignon, en gallo et en normand (avec des formes en /i/), même si, partout, certains locuteurs préfèrent le passé composé - comme du reste en alsacien et en francique pour exprimer l'imparfait français. À l'imparfait, les verbes du $1^{\text {er }}$ groupe dégagent un $/ \mathrm{v} /$ en corse, en catalan, en occitan et en francoprovençal (un $/ \mathrm{v} /$ réalisé $[\mathrm{w}]$ dans certaines variétés de corse et de gascon) : on a ainsi des formes comme avançava - ou avinchive en francoprovençal pour traduire «avançait». Ces imparfaits sont en [0]/[o] dans certaines variétés de bourguignon, de champenois et de picard (ex. avinchot en picard rouchi, parlé dans la région de Valenciennes). À la $3^{\mathrm{e}}$ personne du pluriel, on a un yod et/ou une nasalisation en poitevin-saintongeais et dans le nord du Croissant

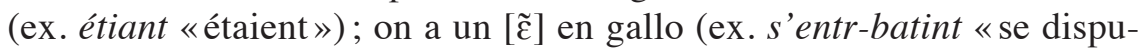
taient»), en angevin, en champenois, en franc-comtois, en bourguignonmorvandiau, en berrichon et en nivernais (parmi les parlers centraux).

Au niveau lexical, le nom de la bise (mot d'origine germanique désignant un vent du nord), comme dans la carte 133 de l'ALF, a donné lieu à un grand nombre d'équivalents qui sont consignés dans le tableau $2:$ dans les parlers de l'Ouest, par exemple, «vent de galerne», «vent frais/froid», «vent d'amont», etc. Par manque de place, nous n'avons pas reproduit de tels tableaux pour d'autres mots comme soleil ou voyageur, mais on peut aisément se rapporter à l'atlas pour faire les correspondances. 


\begin{tabular}{|c|c|}
\hline alsacien & $\begin{array}{l}\text { Bisswìnd, Nìderwìnd, Nordwend, Nordwìnd, } \\
\text { Wind, Wìnd }\end{array}$ \\
\hline angevin & vent agapi, vent fre' \\
\hline basque & aize beltza, ipar haizea, iparraizea \\
\hline bourguignon-morvandiau & bige, bîje, bïlle, bise \\
\hline breton & avel (-viz) \\
\hline catalan & bispa \\
\hline champenois & bise, vo glacé d'hiver \\
\hline corse & $\begin{array}{l}\text { muntagnolu, tramuntana, trattu, trettu, } \\
\text { ventulellu }\end{array}$ \\
\hline flamand occidental & Noordsche wind \\
\hline franc-comtois & bije, bise \\
\hline francique & $\begin{array}{l}\text { énnescht Loft, Kàlde Nordwìnd, Nordwìnd, } \\
\text { Wand vum Norden }\end{array}$ \\
\hline francoprovençal & bisa, bise, bisi, bize, sezante \\
\hline gallo & bise, (petit) vent, vent fret \\
\hline lorrain roman & bihhe \\
\hline mainiot & bise, vent (vent d'à bas) \\
\hline normand & $\begin{array}{l}\text { bise, bllase, hâle, rible, vent d'amont, vent } \\
\text { freid }\end{array}$ \\
\hline occitan & $\begin{array}{l}\text { aire, aura (de bisa), bisa, cerç, cisampa, } \\
\text { essire, mistrau, vent (dau nòrd), vent de } \\
\text { denaut, zisola }\end{array}$ \\
\hline parlers centraux & vent (fou), abisouée \\
\hline picard & bise, vint (d'amont/d'froèdure) \\
\hline poitevin-saintongeais & $\begin{array}{l}\text { bise, ébisall, galerne, vent (d'bise, dau nord, } \\
\text { de Galérne, fret, refredzi) }\end{array}$ \\
\hline wallon & biche \\
\hline
\end{tabular}

Tableau 2. - Traductions du mot bise.

Le verbe bouf(f)er pour «souffler» se retrouve, sous diverses formes, en catalan, dans tout le domaine occitan, dans une grande partie du domaine d'oïl) et même en basque (buhatzen, où on reconnaît le « $\mathrm{h} » \mathrm{du}$ gascon). On a également, dans les parlers de l'Ouest, des formes comme acert( $a$ ) inant pour «assurant» en normand, en mainiot et en poitevin-saintongeais, d'assent pour «d'accord» et hardes pour «manteau» en normand, en gallo et en poitevin-saintongeais (jusque dans la Charente limousine), décapeler en normand et en poitevin-saintongeais. Pour dire «s'est mise», le prétérit 
se botèt que l'on a dans différentes variétés d'occitan trouve son pendant, au passé composé, dans le franc-comtois (ou le lorrain, orthographié différemment) s'ât botèe. Pour dire «briller», l'infinitif lusir que l'on a dans différentes variétés d'occitan trouve son pendant dans le bourguignon ou le lorrain lure. Pour dire «réchauffé», des formes du type réchandu sont partagées par des locuteurs de Bourgogne et du Bourbonnais (dans le Croissant), des formes du type réchalé par des locuteurs d'Anjou, de Normandie et du Poitou. Pour dire «ôté» (<OBSTARE), un préfixe en $r$ - apparaît en lorrain et en franc-comtois (rôtè), tandis que c'est un préfixe en $d$ - (dostat) que l'on trouve dans plusieurs variétés d'occitan : ces deux types sont réunis dans le Französisches etymologisches Wörterbuch [FEW, 7 : 288] (Wartburg, 1922-2002). Pour dire «regardé», enfin, ce sont des variantes de raivoétie (franc-comtois) et de r'ouêti (lorrain) qui, liées à «reguetter», d'origine germanique (*WAHTA), font écho à l'occitan gaitat [FEW, 17 : 456a].

D'autres traits grammaticaux sont propres à certains dialectes, même s'ils ne sont pas étendus à l'ensemble des domaines correspondants. Citons :

- en lorrain, des formes d'imparfait dit prochain, en -z'ôre (Rohlfs, 1966);

- en picard, l'article défini masculin singulier ech;

- en wallon, l'article défini masculin et féminin singulier $l i$;

- en champenois, l'article défini masculin singulier $l u$;

- en poitevin-saintongeais, les pronoms sujets de $3^{\text {e }}$ personne neutre singulier $o$ (ou ol devant voyelle) ainsi que masculin singulier et pluriel le (ou $l$ ' devant voyelle), par exemple dans le bufét («il soufflait», en parlant du vent);

- en gascon, le que explétif, les pronoms eth et $a c$;

- en provençal, des formes plurielles comme dei.

Nous ne nous étendrons pas sur ces traits, qui ont pu faire l'objet d'une importante documentation dialectologique (Poplineau, 2006; Chevrier \& Gautier, 2002; Sumien, 2007, inter alia). La tendance à cultiver la différence est si répandue chez les observateurs comme chez les locuteurs, affirmant que dans telle commune on ne parle pas comme dans la commune voisine voire le hameau d'à côté, qu'il nous a paru plus intéressant de souligner certains points communs. La fable d'Ésope «La bise et le soleil», on l'a vu, est assez riche pour mettre en évidence nombre de phénomènes, malgré sa brièveté. Elle permet en outre au grand public de se repérer, puisqu'il suffit d'écouter la version en français (de Paris) ou suffisamment de variétés de langues romanes (voire germaniques si l'on est germanophone) pour comprendre le contenu de l'histoire. Elle ne permet 
pas, en revanche, d'étudier de façon systématique la négation, la formation du pluriel des noms ou la $1^{\text {re }}$ personne du pluriel des verbes utilisés avec le pronom je. Il faudrait collecter, pour cela, un corpus plus important et poursuivre notre voyage à travers les langues régionales de France.

\subsection{Transcriptions}

Une transcription orthographique du matériel enregistré était cruciale dans notre projet, dans la mesure où celui-ci est destiné au grand public. Comme esquissé plus haut, on est conscient des problèmes que cela pose : problèmes de la normalisation (ou, si l'on préfère, de la «normativisation») linguistique, de la distinction entre description et prescription, etc. Ces problèmes sont bien connus pour une langue comme le corse, précisément à l'origine du concept de langue polynomique - tolérante vis-à-vis de la variation (Marcellesi, 1987). On a ainsi, dans notre texte, des formes comme viaghjatore, viathjatore, viathjatori en corse.

Nous avons tâché de prendre en compte toute la diversité et la complexité aussi bien graphique que sonore des aires linguistiques considérées, afin que les transcriptions orthographiques de l'atlas linguistique mis en ligne soient correctement interprétées par rapport aux orthographes en usage. Face à cette complexité, la conception occitaniste d'une norme à plusieurs niveaux (Alibert, 1935; Sumien, 2007) permet de gérer intelligemment la variation géographique et les pratiques locales. La norme alibertine, prolongé par le Conselh de la Lenga occitana (CLO), privilégie la composante étymologique, à côté de règles à valeur phonologique; tout en étant de base languedocienne, elle admet des usages fréquents dans différentes variétés occitanes.

La norme du CLO a inspiré, entre autres, des militants de l'arpitan (nom que ceux-ci ont donné au francoprovençal ${ }^{12}$ ), sans toutefois que leur Orthographe de référence $\mathrm{B}[\mathrm{ORB}]$ ne remporte l'adhésion de la majorité des locuteurs - en tout cas ceux que nous avons interviewés, rebutés par la nécessité de l'apprentissage d'un système supradialectal. Le francoprovençal est depuis trente ans transcrit, côté français, avec une graphie largement inspirée de celle de Conflans, qui recommande de n'écrire que ce qu'on prononce et d'utiliser, si possible, un système proche de l'ortho-

12. Le glossonyme «francoprovençal», il est vrai, n'est pas satisfaisant : il est trop long et, même sans trait d'union, peut faire croire à un mélange de français et de provençal. Nous le reprenons cependant, car il est le plus consensuel (Martin, 2011). Voir aussi l'étude «Francoprovençal et occitan en Rhône-Alpes» (FORA), <http:// icar.univ-lyon2.fr/projets/ledra/documents/Etude_FORA_rapport_définitif.pdf>. 
graphe française (Martin, 2011). Ce point de vue oralisant rejoint d'autres conventions selon lesquelles la graphie utilisée devrait rendre la lecture facile, être déterminée par la prononciation et laisser de côté les questions grammaticales et étymologiques, tout en respectant les exigences scientifiques des linguistes: on peut ainsi avoir des transcriptions comme kou pour «coup». En pratique, néanmoins, que la motivation soit linguistique ou idéologique (affirmer l'identité de la langue régionale par rapport au français), on est vite en butte à un problème de cohérence : coexistence de $\langle\mathrm{k}$ » et de $\langle\mathrm{qu} »$, de « $\mathrm{z}$ » et de $\langle\mathrm{s}$ » intervocalique, par exemple. On retrouve, du reste, ces problèmes dans d'autres graphies d'inspiration plus phonétique ou des graphies francisantes.

Dans les parlers d'oïl, on trouve des hésitations :

- entre les terminaisons -in, -int et -ain pour les imparfaits de $3^{\mathrm{e}}$ personne du pluriel en $/ \tilde{\varepsilon} /$ (en angevin et dans les parlers centraux);

- entre les terminaisons - $(d)$ jou et - geou $(x)$ pour traduire le mot « voyageur» (en lorrain et en franc-comtois);

- entre deus et deux (en normand et en picard).

L'occitan n'est pas en reste, où l'on peut trouver dans un même dialecte (auvergnat, vivaro-alpin, etc.) des formes comme celles qui suivent, sans que la distinction soit toujours très audible :

- sorelh, solelh, soleu et solèu pour «soleil»;

- mantel, mantèl, manteu, mantèu et mantèlh pour «manteau»;

- disputavan, disputaven et disputaven pour «disputaient»;

- arrivariá, arribariá, arribaria (en occitan alpin, censé être accentué sur le dernier [i]), arribaré (en gascon, au futur dans le passé) pour «arriverait»;

- promier, premier, premièr, primier, primièr, prumier, prumièr pour «premier»;

- bofar/bofava et bufar/bufava pour «souffler»/«soufflait» (y compris chez un même locuteur);

- alòr et alòrs (forme également socialisé) pour «alors »;

- dels, deus et daus pour «des».

On a affaire, on le constate, à nombre de problèmes provenant de la présence ou de l'absence d'un diacritique. L'utilisation de l'accent graphique, qui marque un accent tonique en occitan (alors que la place de l'accent tonique n'a pas beaucoup de sens dans la grande majorité des parlers du Croissant), permet d'indiquer des distinctions d'aperture vocalique 
dans ce contexte. Cette distinction, cependant, n'est pas toujours très nette, notamment en limousin, où elle a pu se perdre (Desrozier \& Ros, 1974). De nouveau, on se heurte à une double difficulté : combiner la transparence graphie-phonie et la correspondance entre les différentes variétés. Il n'en demeure pas moins qu'on est loin de l'anarchie graphique qui prévaut dans le domaine d'oïl.

Une estimation de cette anarchie graphique peut être donnée en calculant le nombre moyen d'apostrophes, par variété, dans les transcriptions (cf. fig. 2). Ce nombre va de 8 en gallo et de 14 en poitevin-saintongeais (pour lesquels des standards ont été proposés) à 31 en mainiot et à plus de 50 en angevin (pour lesquels nous disposons essentiellement de graphies personnelles). Il est à titre de comparaison de 5 en français (standard) et en moyenne de 7 en occitan. Ces apostrophes, le plus souvent introduites là où en français on aurait un $e$ muet ou une liquide élidée $(l$ ou $r$ ) sont le signe que ces graphies d'oïl restent en grande partie inféodées aux usages français.

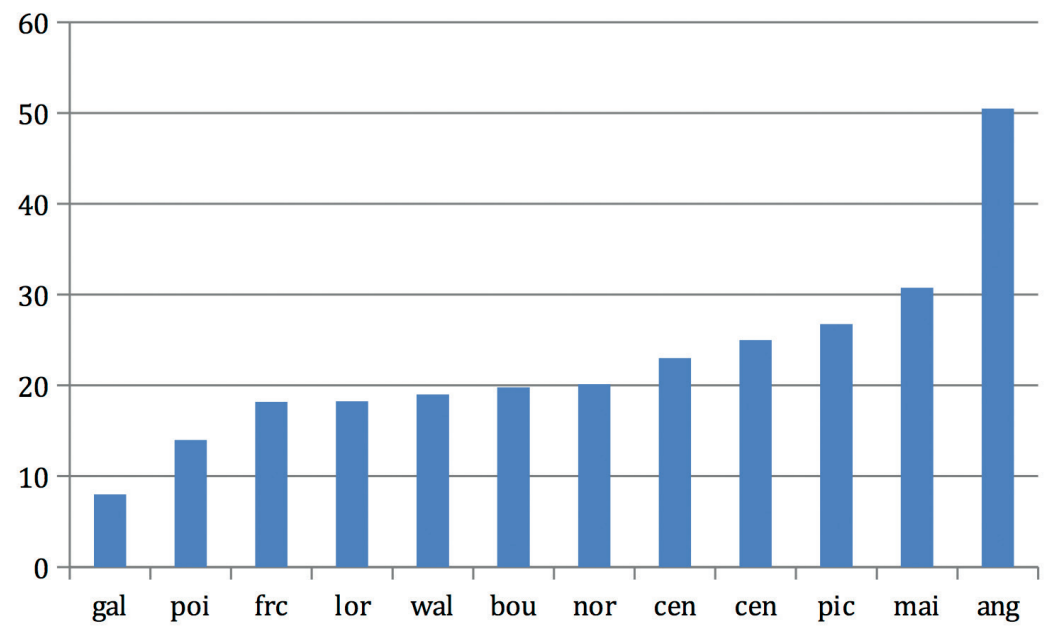

Figure 2. - Nombre moyen d'apostrophes par variété d'oil dans les transcriptions (ordre croissant).

\section{Conclusion et perspectives}

Cet atlas multimédia fournit une sorte de photographie en couleur de l'arcen-ciel dialectal de la France (hexagonale) : un instantané des usages actuels, un outil pour l'enseignement de la variation, montrant que les langues territoriales de France, même menacées de disparition, sont aujourd'hui encore 
une réalité précieuse (D’Hervé, 2005). Il ne nous dit pas grand-chose de la vitalité de ces langues, du nombre de leurs locuteurs ${ }^{13}$, des dynamiques sociales qui sont à l'œuvre, malgré la quantité de données recueillies. Il ne dit pas non plus combien enrichissantes et touchantes ont été les rencontres personnelles avec les informateurs : véritables échanges entre témoins qui souvent se savent les derniers locuteurs des langues qu'ils représentent et linguistes, qui ont beaucoup à apprendre d'eux.

La pratique des langues régionales territoriales s'est raréfiée et est devenue très minoritaire : la chaîne de transmission intergénérationnelle au sein des cellules familiales est souvent interrompue. L'enjeu était donc de taille, pour donner une plus grande visibilité à la diversité du paysage linguistique français. Et les locuteurs auxquels nous avons demandé de traduire une courte fable se sont fort bien acquittés de cette tâche.

La représentativité des locuteurs reste un vrai problème, par rapport à un cadre dialectologique traditionnel qui suppose un lien inamovible entre la terre et le locuteur. Ce cadre est de plus en plus dépassé dans un pays dominé par la mobilité et l'urbanisation (Mufwene \& Vigouroux, 2012). De grandes villes comme Marseille, Toulouse ou Montpellier brassent nécessairement nombre de différences, et l'influence du français sur les langues régionales est aujourd'hui inévitable. Le protocole retenu (une traduction) peut également avoir une influence, incitant à porter un regard critique sur les productions ainsi élicitées. Toutefois, en fonction des locuteurs, la traduction peut favoriser aussi bien des calques qu'une recherche de déviance maximale vis-à-vis du français.

Nous avions à cœur de toucher les linguistes et les enseignants, mais aussi le grand public. Cet objectif a été atteint, au vu du succès médiatique qu'a connu notre site (avec 300000 visites à l'été 2017), dans la presse écrite et audio-visuelle ainsi que dans les réseaux sociaux. Le but était de rattraper le retard pris par notre pays dans la mise en valeur de ses langues minoritaires. Associer ces dernières à la modernité (avec des outils cartographiques innovants pour présenter les limites entre aires dialectales) pourra, espérons-le, contribuer à revitaliser notre héritage linguistique, à lui donner du moins une reconnaissance en tant que vecteur de créativité, à défaut de contrecarrer le déclin des pratiques langagières minoritaires.

13. Nous renvoyons à Héran, Filhon et Deprez (2002) pour une estimation du nombre de locuteurs de langues régionales en France métropolitaine et à Bernissan (2012) plus particulièrement pour l'occitan. Les nombres avancés allant pour l'occitan de 526000 à 110000 , d'une publication à l'autre, on voit les problèmes que posent de telles estimations. 
La valorisation de celles-ci passant notamment par la graphie, nous avons insisté sur ce dernier aspect, dans cet article où nous avons illustré, organisé, analysé et codifié des enregistrements en dialectes et langues de France. Si l'on écrit, c'est essentiellement pour être lu, si possible avec plaisir; et il faut prendre garde à ne pas se couper des usages réels.

N'est-ce pas seulement depuis un peu plus d'un siècle que l'orthographe française s'est figée (Catach, 1992), alors que notre langue peut se prévaloir d'une littérature bien plus ancienne? Les questions d'aménagement linguistique et d'action glottopolitique sont passionnantes, mais dépassent sans doute le cadre de ce travail. Dans cette perspective, les transcriptions des points d'enquête que nous avons présentés ici offrent des pistes concrètes. Les solutions proposées à ce problème du passage à l'écrit, pour des langues moins normées que le français, on l'a vu, sont diverses et relèvent souvent d'idéologies distinctes, parfois opposées, ce qui rend le sujet épineux.

Nous continuerons à donner un écho médiatique à cet atlas sonore, que nous allons étendre aux Outre-mer, afin de le valoriser et d'en faire la publicité. Pour aller plus loin, il est envisageable non seulement d'utiliser la toile pour afficher les fruits de la recherche, mais aussi pour collecter de nouvelles informations, manquantes, selon une méthodologie de crowdsourcing actuellement très en vogue. On pourra, par exemple, demander à nos informateurs de dénommer dans leurs variétés dialectales une centaine de concepts de l'ALF - de mots comme noix, prunelles ou pissenlit. Proposer de compléter des points d'enquête à travers Internet demandera cependant quelques précautions pour les langues minoritaires.

\section{Remerciements}

Ce travail a en grande partie été financé dans le cadre de l'appel à projets «Langues et numérique 2016» de la Délégation générale à la langue française et aux langues de France (DGLFLF). Nous remercions F. Manni et M. Russo pour leur coopération à ce projet, ainsi que G. Brun-Trigaud, qui nous a fourni les latitudes et longitudes de quelque 600 points de l'ALF. Nous tenons à exprimer par-dessus tout notre sincère gratitude envers tous ceux qui ont accepté de nous accorder de leur temps et de prêter leur voix à cette réalisation. Il va sans dire que cet atlas sonore n'aurait pas été possible et n'aurait eu aucun sens sans eux. Nous sommes également très reconnaissants envers les personnes suivantes, qui nous ont aidés à entrer en contact avec les locuteurs et les locutrices : I. Duguine pour le basque, P. Erhart pour l'alsacien, J.-L. Kieffer pour le francique, J.-P. Couché pour le flamand occidental, S. Retali-Medori pour le corse, H. Tyne pour le catalan, 
O. Lalliard pour le francoprovençal, R. Sichel-Bazin pour l'occitan, J. L. Léonard pour le bourguignon-morvandiau, L. Jagueneau pour le poitevinsaintongeais, B. Massot pour le lorrain, S. Bertin pour le mainiot, M. Lebas pour le normand, J.-M. François pour le picard, Y. Guilloux pour les parlers centraux, N. Quint pour le Croissant et tant d'autres, envers lesquels nous sommes redevables à plus d'un titre.

\section{RÉFÉRENCES BIBLIOGRAPHIQUES}

Abalain Hervé, 2007, Le français et les langues historiques de la France, Paris, Éd. Jean-Paul Gisserot.

AliBERT Louis, 1935, Gramatica occitana segon los parlars lengadocians, Toulouse, Societat d'Estudis Occitans.

Bernissan Fabrice, 2012, «Combien de locuteurs compte l'occitan en 2012?», Revue de linguistique romane, $\mathrm{n}^{\circ} 76, \mathrm{p} .467-512$.

Bourcelot Henri, 1966-1978, Atlas linguistique et ethnographique de la Champagne et de la Brie, Paris, Éd. du CNRS.

Brasseur Patrice, 1980-2011, Atlas linguistique et ethnographique normand, Paris, Éd. du CNRS.

CARTON Fernand, 2009, «Pourquoi et pour qui on transcrit? Les graphies du picard moderne », La Linguistique, $\mathrm{n}^{\circ} 45$, p. 113-123.

Carton Fernand \& Lebègue Maurice, 1989-1997, Atlas linguistique et ethnographique de la Picardie, Paris, Éd. du CNRS.

CATACH Nina, 1992, L'orthographe, Paris, Presses universitaires de France.

Caubet Dominique, Chaker Salem \& Sibille Jean, 2002 (éds), Codification des langues de France, Paris, L'Harmattan.

Chambon Jean-Pierre \& Greub Yan, 2002, «Note sur l'âge du (proto)gascon», Revue de linguistique romane, $\mathrm{n}^{\circ} 66, \mathrm{p} .263-264$.

CHevrier Jean-Jacques \& GAUTIER Michel, 2002, Le poitevin-saintongeais : langue d'oül méridionale, La Crèche, Gestes Éditions.

Contejean Charles, 1876, Glossaire du patois de Montbéliard, Montbéliard, Société d'émulation de Montbéliard.

Costaouec Denis, 2013, «Politiques linguistiques : le cadre légal en France», dans J. C. Herreras (éd.), Politiques linguistiques en Europe, Valenciennes, Presses universitaires de Valenciennes, p. 131-157.

D'Hervé Gildas, 2005, «Le gallo dans l'enseignement, l'enseignement du gallo», Marges linguistiques, $\mathrm{n}^{\circ} 10$, p. 262-281.

Desrozier Peir \& Ros Joan, 1974, L'ortografia occitana . Lo lemosin, Montpellier, Centre d'Estudis Occitans.

Dondaine Colette, 1972-1978, Atlas linguistique et ethnographique de la FrancheComté, Paris, Éd. du CNRS.

Dubuisson Pierrette, 1971-1982, Atlas linguistique et ethnographique du Centre, Paris, Éd. du CNRS. 
Gaillard-Corvaglia Antonella, Léonard Jean-Léo \& Darlu Pierre, 2007, «Testing Cladistics on Dialect Networks and Phyla (Gallo-Romance Vowels, Southern Italo-Romance Diasystems and Mayan Languages)», dans 9th Meeting of the ACL SIG in Computational Morphology and Phonology, Prague, p. 23-30.

Gardette Pierre, 1952, «Atlas linguistique et ethnographique du Lyonnais », Revue de géographie de Lyon, vol. 27, n 1, p. 90-92.

GiLliéron Jules \& Edmont Edmont, 1902-1910, Atlas linguistique de la France, Paris, Champion.

Gilliéron Jules \& Mongin Jean, 1905, Étude de géographie linguistique : «scier» dans la Gaule romane du Sud et de l'Est, Paris, Champion.

Goebl Hans, 2002, «Analyse dialectométrique des structures de profondeur de l'ALF», Revue de linguistique romane, vol. 66, n² 261-262, p. 5-63.

Guillaume Gabriel \& Chauveau Jean-Paul, 1975-1983, Atlas linguistique et ethnographique de la Bretagne Romane, de l'Anjou et du Maine, Paris, Éd. du CNRS.

HeEringa Wilbert, 2004, Measuring Dialect Pronunciation Differences Using Levenstein Distance, these de doctorat, Rijksuniversiteit, Groningue (Pays-Bas).

Héran François, Filhon Alexandra \& Deprez Christine, 2002, «La dynamique des langues en France au fil du $\mathrm{xx}^{\mathrm{e}}$ siècle», Population et Sociétés, $\mathrm{n}^{\circ} 376, \mathrm{p} .1-4$.

HudLetT Albert, 2004, Charte de la graphie harmonisée des parlers franciques - platt - de la Moselle germanophone, Mulhouse, Publications du Centre de recherche sur l'Europe littéraire.

IAnNÀCCARo Gabriele \& Dell'Aquila Vittorio, 2001, «Mapping Languages from Inside: Notes on Perceptual Dialectology », Social \& Cultural Geography, $\mathrm{n}^{\circ} 2-3$, p. 265-280.

JACobson Michel \& BAude Olivier, 2011, «Corpus de la parole : collecte, catalogage, conservation et diffusion des ressources orales sur le français et les langues de France», Traitement automatique des langues, vol. 52, n 3, p. 47-69.

JoRet Charles, 1881, Essai sur le patois normand du Bessin : suivi d'un dictionnaire étymologique, Paris, F. Vieweg.

LANHER Jean, LitAize Alain \& Richard Jean, 1979-1988, Atlas linguistique et ethnographique de la Lorraine romane, Paris, Éd. du CNRS.

Le Dû Jean, Le Berre Yves \& Brun-Trigaud Guylaine, 2005, Lectures de l'atlas linguistique de la France de Gilliéron et Edmont. Du temps dans l'espace, Paris, CTHS.

LÉONARD Jean-Léo \& JAGUENEAu Liliane, 2013, «Disparition, apparition et réapparition des langues d'oïl : de l'invisibilisation au nouveau regard», Bulletin de la Société de linguistique de Paris, vol. 108, n 1, p. 283-343.

MarCellesi Jean-Baptiste, 1987, «L'action thématique programmée : “individuation sociolinguistique corse" et le corse polynomique», Études corses, n 28 , p. 5-20.

MARquet Isabelle, 1995, Atlas linguistique parlant d'une région des Alpes occidentales. Étude de la variabilité phonétique entre le Vercors et la frontière italienne, thèse de doctorat, Université Stendhal-Grenoble 3. 
Martin Fanny, 2015, Espaces \& lieux de la langue au XXI siècle en Picardie : approche complexe de la structuration des pratiques linguistiques en situations ordinaires. Enquêtes en Picardie, thèse de doctorat, Université de Picardie Jules Verne, Amiens.

Martin Jean-Baptiste, 2011, «Le francoprovençal », Langues et Cité, n 18, p. 1-12.

Massignon Geneviève \& Horiot Brigitte, 1971-1983, Atlas linguistique et ethnographique de l'Ouest, Paris, Éd. du CNRS.

MEdori Stella, 2013, «Éléments gallo-italiens et gallo-romans dans les parlers corses », Revue de linguistique romane, $\mathrm{n}^{\circ} 305-306$, p. 121-138.

MéDÉLICE Jeanine Élisa, 2008, «Présentation du projet de l'Atlas linguistique multimédia de la Région Rhône-Alpes et des zones limitrophes (ALMURA) et commentaires du poster», dans G. Raimondi et L. Revelli (éds), Dove va la dialettologia?, Alexandrie, Edizioni dell'Orso, p. 199-205.

Mufwene Salikok S. \& Vigouroux Cécile B., 2012, «Individuals, Populations, and Timespace: Perspectives on the Ecology of Language», Cahiers de sociolinguistique, vol. 38, $\mathrm{n}^{\circ} 2$, p. 111-137.

PARIS Gaston, 1888, «Les parlers de France», Revue des patois gallo-romans, $\mathrm{n}^{\mathrm{o}} 17, \mathrm{p} .161-175$.

Poplineau Bernard, 2006, «Méthode d'apprentissage du champenois », Lou Champaignat, $\mathrm{n}^{\circ} 28$, p. 5-31.

Rispail Marielle, 2014, «Une enquête en pays francique», Langues et Cité, $\mathrm{n}^{\circ} 25$, p. $10-11$.

RoHLFs Gerhard, 1966, «Welsches Patois im Elsass», Zeitschrift für französische Sprache und Literatur, $\mathrm{n}^{\circ} 76$, p. 269-270.

SÉGuY Jean, 1973a, «Les Atlas linguistiques de la France par régions », Langue française, $\mathrm{n}^{\circ} 18$, p. 65-90.

SÉGUY Jean, 1973b, «La dialectométrie dans l'Atlas linguistique de la Gascogne», Revue de linguistique romane, $\mathrm{n}^{\circ} 37$, p. 1-24.

Simoni-Aurembou Marie-Rose, 1973-1978, Atlas linguistique et ethnographique de l'Île-de-France et de l'Orléanais, Paris, Éd. du CNRS.

Sumien Domergue, 2007, «Preconizacions del Conselh de la Lenga Occitana», Lingüistica Occitana, $\mathrm{n}^{\circ}$ 6, p. 1-157.

Sumien Domergue, 2008, «Classificacion dei dialèctes occitans », Linguistica Occitana, $\mathrm{n}^{\circ} 7, \mathrm{p} .1-55$.

Sumien Domergue, 2012, «Le catalogue des langues romanes : clarifier les critères et les idéologies », Revue des langues romanes, vol. 116, $\mathrm{n}^{\circ}$ 1, p. 5-33.

TAVERDET Gérard, 1975-1980, Atlas linguistique et ethnographique de la Bourgogne, Paris, Éd. du CNRS.

TuAillon Gaston, 1976, Comportement de recherche en dialectologie française, Paris, Éd. du CNRS.

Tuaillon Gaston \& Contini Michel, 1996, Atlas linguistique roman (AliR), Rome, Istituto poligrafico e zecca dello stato.

VANnini Laurent \& Le Crosnier Hervé, 2012, Net.lang : réussir le cyberespace numérique, Caen, C\&F Éditions. 
WARTBURG Walter vON, 1922-2002, Französisches etymologisches Wörterbuch: eine Darstellung des galloromanischen Sprachschatzes, Bonn/Leipzig/Bâle, Teubner/Klopp/Zbinden.

Zeidler Edgar \& Crévenat-Werner Danielle, 2008, Orthographe alsacienne : bien écrire l'alsacien de Wissembourg à Ferrette, Colmar, J. Do Bentzinger. 